\title{
Dark Matter Searches
}

\author{
K. Pretzl
}

Received: 26 December 2006 / Accepted: 1 February 2007 /

Published online: 23 March 2007

(C) Springer Science+Business Media, Inc. 2007

\begin{abstract}
According to our present knowledge the matter/energy budget of the universe consists of $74 \%$ dark energy, $22 \%$ dark matter and $4 \%$ ordinary (or so-called baryonic) matter. While the dark energy cannot be detected directly, searches for dark matter are performed with earth-bound and space-borne detection devices, assuming that the dark matter consists of weakly interacting massive particles, the so-called WIMPs. An overview of the present experimental situation is given.
\end{abstract}

Keywords Dark matter

\section{Introduction}

Since the discovery of dark matter in the Coma cluster by the Swiss astronomer Fritz Zwicky (1933) 73 years have passed and we still do not know what the real nature of it is. Dark matter shows its presence by gravitational interaction with ordinary matter. It holds numerous galaxies together in large clusters and it keeps stars rotating with practically constant velocities around the centers of spiral galaxies. With gravitational lensing, a technique which was originally proposed by Fritz Zwicky (1937) to determine the mass of galaxies and galaxy clusters, we are able to obtain today a very good picture of how the dark matter is distributed in the universe. We believe that the dark matter played an essential role during the formation of the galaxies and galaxy clusters. The dark matter provided the gravitational wells into which ordinary matter was drawn during the early evolution of the universe.

Recently much information about the matter/energy content of the universe was gained from several independent observations like: The Cosmic Microwave Background radiation (CMB), the Large Scale Structure surveys (LSS), the cluster searches and the Super Novae type 1a surveys (SN) (Spergel 2006). Combining the results of these observations the matter/energy budget of the universe turns out to be: $74 \pm 2 \%$ dark energy, $26 \pm 2 \%$ matter

\section{K. Pretzl ( $\square)$}

Laboratory for High Energy Physics, University of Bern, Bern, Switzerland

e-mail: pretzl@lhep.unibe.ch 
(including baryonic and dark matter) and $4.4 \pm 0.3 \%$ baryonic matter. It is quite remarkable that similar baryonic matter densities could be derived from the light element abundances and the nuclear synthesis, to which Johannes Geiss and collaborators contributed very significantly. It was one of the main topics at this symposium (see contributions of J. Geiss, N. Prantzos, Th. Bania and H. Reeves).

The fact that $96 \%$ of the universe is of unknown nature provides enough motivation and challenge for scientists of different fields like astronomy, astrophysics, cosmology and particle physics to search for dark matter and dark energy in earth-bound and space-borne experiments. This article will concentrate on dark matter searches and will not report on developments to disentangle the mystery of the dark energy, which seems to be related to Einstein's cosmological constant. However, there are not many hints about the true nature of the dark matter other than it interacts gravitationally with ordinary matter and it must have been produced in an early phase of the universe. It may, however, also interact via very weak and so far unknown forces with matter. Still, particle physicists proposed some possible candidates:

1. Massive neutrinos. They would be natural candidates since they are among the most abundant particles in the universe. Neutrinos with a mass of a few $\mathrm{eV} \mathrm{c}^{-2}$ would significantly contribute to the missing mass. Since neutrinos are relativistic when they decouple from matter, they qualify as hot dark matter (HDM) candidates.

2. Weakly interacting massive particles, the so-called WIMPs. The most favored candidate is the neutralino, which is a particle predicted by Super Symmetry (SUSY). SUSY is the best studied extension of the Standard Model (SM) of particle physics. If the neutralino existed two problems could find a solution at the same time, namely the dark matter and the physics beyond the SM. Neutralinos would be candidates for cold dark matter (CDM) since they would be heavy and nonrelativistic when they decouple from matter in the early universe.

3. Axions. These particles, named after a laundry detergent, are associated with a pseudoscalar field, which was introduced by Peccei and Quinn (1977) to solve the charge conjugation and parity $(\mathrm{CP})$ violation problem in Quantum Chromodynamics (QCD), the theory of strong interactions. Axions would be produced abundantly during the QCD phase transition in the early universe when hadrons were formed from quarks and gluons. Since axions are nonrelativistic at freeze out they would qualify as CDM candidates.

The direct detection of dark matter, if it exists in form of particles, is encouraged by the large expected particle flux which can be deduced under the following assumptions. In an isothermal dark matter halo model the velocity of particles in our galaxy is given by a Maxwell Boltzmann distribution with an average value of $\langle v\rangle=230 \mathrm{~km} \mathrm{~s}^{-1}$ and an upper cutoff value of $575 \mathrm{~km} \mathrm{~s}^{-1}$ corresponding to the escape velocity. The dark matter halo density in our solar neighborhood is estimated to be $\rho=0.3 \mathrm{GeV} \mathrm{c}^{-2} \mathrm{~cm}^{-3}$. From that one expects a flux of $\Phi=\rho\langle v\rangle / m_{\chi} \sim 7 \times 10^{6} / m_{\chi} \mathrm{cm}^{-2} \mathrm{~s}^{-1}$ with $m_{\chi}$ the mass of the dark matter particle in $\mathrm{GeV} \mathrm{c}^{-2}$. However, since neither the mass nor the interaction cross section of these particles are known one is forced to explore a very large parameter space, which requires very sensitive and efficient detection systems. In the following article some of the more recent dark matter searches are described.

\section{Neutrinos, Do They Qualify for Dark Matter Candidates?}

In most Big Bang models it is assumed that the relic abundance of neutrinos is comparable (9/11) to that of photons. Neutrinos come in 3 flavors: the electron neutrino $v_{e}$, the muon 
neutrino $v_{\mu}$ and the tau neutrino $v_{\tau}$. They contribute with $\Omega_{v} \cdot h^{2}=\sum m_{v} / 94 \mathrm{eV} \mathrm{c}^{-2}$ to the matter density of the universe, where $\Omega_{v}$ is the neutrino density normalised to the critical density of the universe, $\mathrm{h}$ the Hubble parameter and $\sum m_{v}$ is the sum over the three neutrino masses in $\mathrm{eV} \mathrm{c}^{-2}$. This illustrates that neutrinos with a mass of a few $\mathrm{eV} \mathrm{c}^{-2}$ could make up most of the missing dark mass. We know from neutrino oscillation experiments that neutrinos must have a rest mass, but the absolute mass values are still missing. Direct neutrino mass measurements have so far resulted only in upper limits: $\leq 2.2 \mathrm{eV} \mathrm{c}^{-2}$, $\leq 160 \mathrm{keV} \mathrm{c}^{-2}, \leq 18 \mathrm{MeV} \mathrm{c}^{-2}$ for the electron, the muon and the tau neutrino respectively. From neutrino oscillation experiments with atmospheric, solar, reactor and accelerator neutrinos (Super-Kamiokande, SNO, KamLAND, K2K and MINOS) (Maltoni et al. 2004) only mass differences can be extracted. The combined results of these experiments yield mass differences for $v_{e} \rightarrow v_{\mu}$ oscillations of $\Delta m^{2}=(8.1 \pm 0.3) \times 10^{-5} \mathrm{eV}^{2} \mathrm{c}^{-4}$ and assuming $v_{\mu} \rightarrow v_{\tau}$ oscillations $\Delta m^{2}=(2.3 \pm 0.6) \times 10^{-3} \mathrm{eV}^{2} \mathrm{c}^{-4}$ leaving open the question of the mass hierarchy. Assuming a mass hierarchy for neutrinos similar to that of charged leptons which is inspired by Grand Unified Theories (GUT) the heaviest neutrino would have a mass of the order of $\leq 0.1 \mathrm{eV} \mathrm{c}^{-2}$. In this case neutrinos would very insignificantly contribute to the missing mass in the universe.

An upper limit for the sum of the neutrino masses can be obtained, when combining the results of CMB, LSS and SN experiments. Neutrinos being relativistic at freeze out are free streaming particles, which cluster preferentially at very large scales. Therefore massive neutrinos would enhance large-scale and suppress small-scale structure formations. From HDM and CDM model calculations fitting the LSS power spectrum one obtains a value for the ratio neutrino density to matter density $\Omega_{v} / \Omega_{m}$. From this value and $\Omega_{m}$ obtained from $\mathrm{CMB}$ and SN an upper limit for the sum of the neutrino masses $\sum m_{v} \leq 0.68 \mathrm{eV} \mathrm{c}^{-2}$ can be derived (Spergel 2006). It is interesting to note that from cosmology we have the best upper limit for the neutrino masses obtained so far. It strongly indicates, however, that neutrinos do not qualify for the dark matter. Model calculations using CDM and a cosmological constant $\Lambda$ for the dark energy $(\Lambda \mathrm{CDM})$ seem to be in overall good agreement with the data.

\section{Weakly Interacting Massive Particles, So-Called WIMPs}

SUSY predicts the existence of new particles, of which the lightest and most stable can be a candidate for CDM. However, SUSY was not invented in the first place to solve the dark matter problem. Its main aim is to unify all forces in the universe in a GUT theory. SUSY predicts bosonic partners to the well known leptons and quarks, which are fermions, and fermionic partners to the known gauge bosons, which are transmitting the forces. If SUSY exists in nature, new particles and new forces would have to be discovered. The SUSY particle which is mostly favored as a candidate for the dark matter is the neutralino, which is neutrally charged as its name already indicates. In the Minimal Super Symmetric Model (MSSM) the neutralino is a linear superposition of four neutral spin 1/2 gauginos:

$$
\chi=n_{11} \tilde{W}^{3}+n_{12} \tilde{B}+n_{13} \tilde{H}_{1}+n_{14} \tilde{H}_{2}
$$

with $\tilde{W}^{3}$ and $\tilde{B}$, the Wino and Bino, and $\tilde{H}_{1}$ and $\tilde{H}_{2}$, the two Higgsinos. Because of its complex structure the parameter space is large and makes mass and interaction cross section predictions difficult. However, the parameter space was constrained recently by the results from the electron positron collider (LEP) at CERN and from the measurements of the anomalous magnetic moment of the muon at Brookhaven. Within the Constrained Minimal 
SUSY Model (CMSSM) the best estimate of the neutralino mass lies between $50 \mathrm{GeV} \mathrm{c}^{-2}$ and $600 \mathrm{GeV} \mathrm{c}^{-2}$ (Ellis et al. 2000). This mass range is well accessible to the Large Hadron Collider (LHC) at CERN, which is expected to be operational in 2007. The search for SUSY particles will be amongst the most prominent subjects for the LHC. In case neutralinos are first found at the LHC, they still need to be confirmed as the dark matter in the universe by their direct detection in WIMP experiments.

\section{Direct Detection of Dark Matter}

The direct detection of WIMPs is based on the measurement of nuclear recoils in elastic WIMP scattering processes. In the case of neutralinos, spin-independent coherent scatterings as well as spin-dependent scatterings are possible. The expressions for the corresponding cross sections can be found in Jungman (1996) and Pretzl (2002). In order to obtain good detection efficiencies, devices with high sensitivity to low nuclear recoil energies $(\mathrm{eV})$ are needed. WIMP detectors can be categorized in conventional and cryogenic devices. Most of the conventional WIMP detectors use NaI, Ge crystals, liquid Xenon (LXe) or liquid Argon (LAr). Conventional devices have the advantage that large detector masses ( $\sim$ ton) can be employed, which makes them sensitive to annual modulations of the WIMP signal owing to the movement of the earth with respect to the dark halo rest frame. Annual modulation, if observed, would provide strong evidence for a WIMP signal, assuming it is not faked by spurious modulated background signals. However, due to quenching of the ionization signals, conventional detectors have lower nuclear recoil detection efficiencies than cryogenic devices.

Cryogenic detectors are able to measure small recoil energies with high efficiency because they measure the total deposited energy in form of ionization and heat (phonons). A small energy loss $\Delta \mathrm{E}$ can lead to an appreciable temperature increase $\Delta T=\Delta E / C$, provided the detector is operated at low enough temperatures (typically $\mathrm{mK}$ ), where the heat capacity $C$ is small. A description of the various detection principles can be found in Pretzl (2000). Cryogenic detectors are made of many different materials, like $\mathrm{Ge}, \mathrm{Si}$, $\mathrm{TeO}_{2}$, sapphire $\left(\mathrm{Al}_{2} \mathrm{O}_{3}\right), \mathrm{LiF}, \mathrm{CaWO}_{4}$ and $\mathrm{BGO}$, including superconductors, like Sn, $\mathrm{Zn}$, $\mathrm{Al}$, etc. This turns out to be an advantage for the WIMP search, since the resulting recoil spectra are characteristically different for detectors with different materials, a feature which helps to effectively discriminate a WIMP signal against background. In comparison to conventional detectors, however, cryogenic detectors are rather limited in target mass $(\sim \mathrm{kg})$. The most frequently used cryogenic devices are bolometers, which consist of an absorber and a sensitive thermometer attached to it. Another technique uses superheated superconducting granules (SSG). An SSG WIMP detector (ORPHEUS) consists of billions of spherical Sn granules with diameters of about $30 \mu \mathrm{m}$. The detector is operated in a magnetic field. WIMPs interacting in a granule can cause a phase transition from the superconducting to the normal state. This phase transition of individual granules can be detected by pickup loops which measure the flux change due to the disappearance of the Meissner-Ochsenfeld effect. The energy threshold of the detector is adjustable by setting the external magnetic field just below the phase transition boundary.

WIMP detectors are located in underground laboratories so as to be protected from cosmic ray background. In addition to a passive shielding against radioactivity from surrounding walls they need to be built from radio poor materials. This shielding alone provides only limited effectiveness and is expensive. Nevertheless, cryogenic bolometers, as used 


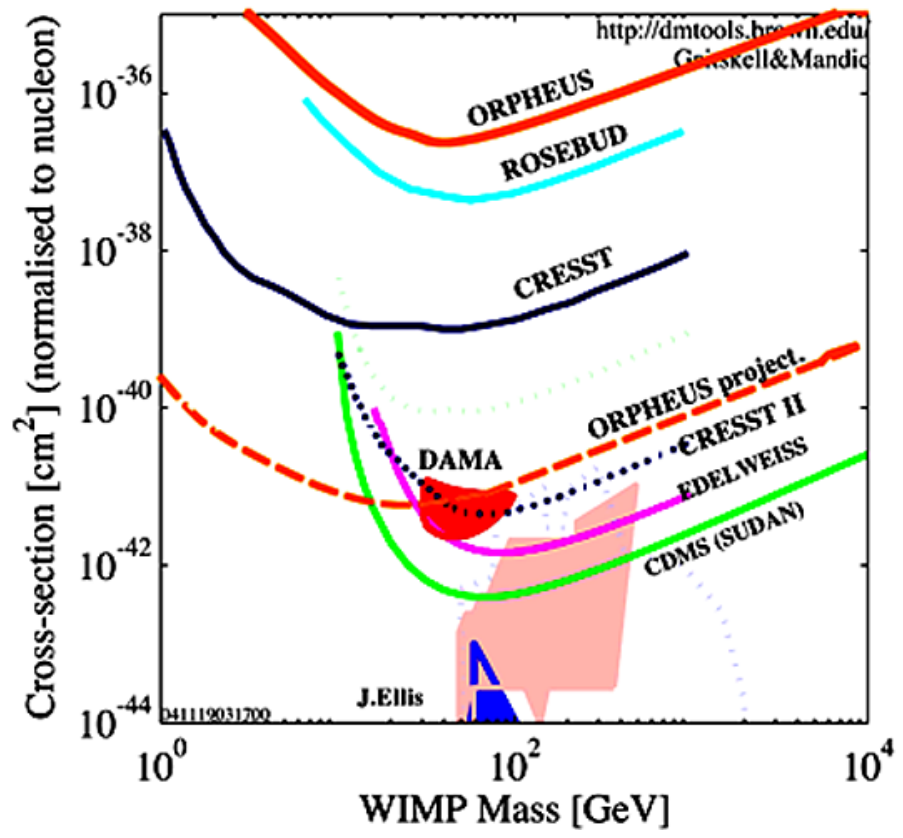

Fig. 1 Exclusion plot for spin-independent WIMP interactions

by CDMS, EDELWEISS and CRESSTII, are able to distinguish nuclear recoils from minimum ionizing particles (Compton electrons) by measuring heat and ionization or photons, in the case of scintillating absorbers, for each event simultaneously, but separately. For the same deposited recoil energy, the ionization (or photon) signal from nuclear recoils is highly quenched compared to signals from electrons, a feature which allows to separate genuine nuclear recoils from electron background. This method turns out to be a very effective active background rejection. An active background rejection was also practiced with the ORPHEUS SSG detector, since minimum ionizing particles cause many granules to flip, while WIMPs cause only one granule to flip (flip meaning a transition from the superconducting to the normal state). More conventional detectors (NaI, LXe, LAr) rely on a signal shape analysis to reduce the background, which turns out to be less powerful.

The experimental results are usually presented as exclusion plots, which show the WIMPnucleon cross section versus the WIMP mass. The exclusion plots from different experiments are shown in Fig. 1 for spin independent WIMP interactions (90\% C.L.) and in Fig. 2 for spin dependent interactions.

So far the lowest cross section limits on spin independent interactions were obtained with cryogenic detectors like: CDMS (absorber: $1 \mathrm{~kg} \mathrm{Ge}, 0.2 \mathrm{~kg} \mathrm{Si}$ ) at Soudan (2090 m.w.e.) (Akerib 2005), EDELWEISS (1 kg Ge) at Frejus (4800 m.w.e.) (Sanglard 2005) and CRESSTII $\left(0.6 \mathrm{~kg} \mathrm{CaWO}_{4}\right)$ at Gran Sasso (3800 m.w.e.) (Angloher 2005). The results of these experiments are not in agreement with the DAMA (100 kg NaI) experiment, which claimed to see an annual modulation signal consistent with a WIMP with a mass $m_{x}=\left(52_{-8}^{+10}\right) \mathrm{GeV} \mathrm{c}^{-2}$ (Bernabei 2000). Also shown in are the results of the first phase of the ORPHEUS (SSG, $0.2 \mathrm{~kg} \mathrm{Sn}$ ) experiment at the Bern underground laboratory (70 m.w.e.) (Borer 2004). With the same absorber mass, but some improvements on the detector and the shielding, limits as shown by ORPHEUS projected in Fig.1 can be reached in future. The main advantage 


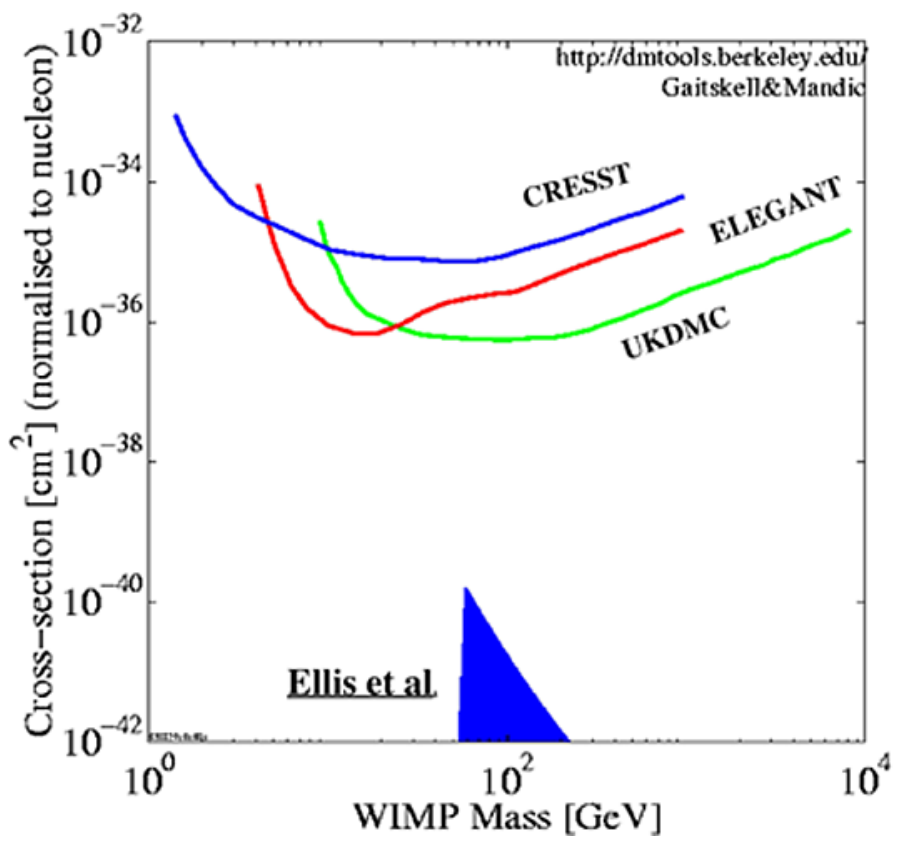

Fig. 2 Exclusion plot for spin-dependent WIMP interactions

of SSG compared to bolometers is that they are potentially more sensitive to low WIMP masses due to the intrinsically lower recoil energy thresholds they can apply.

Exclusion plots for spin-dependent WIMP interactions from the experiments CRESST (0.262 kg Al $\mathrm{O}_{3}$ ) (Angloher 2002), ELEGANT (660 kg NaI) at Kamioka (2700 m.w.e.) (Yoshida 2000) and UKDMC (6 kg NaI) at Boulby Mine (2530 m.w.e.) (Bravin 1999) are shown in Fig. 2. CDMS (Akerib 2006) has recently published upper limits for spindependent WIMP interaction cross sections normalized to protons and neutrons separately, which turn out to be slightly lower than the one shown in Fig. 2. The CMSSM model predictions of Ellis et al. (2000), also shown in Fig. 1 and Fig. 2, are still several orders of magnitude below the presently reached experimental sensitivities. However, a new generation of cryogenic WIMP detectors planning to employ absorber masses of 10-100 kg and conventional detectors, like GENIUS (Ge), with a mass of 1 ton are in preparation in order to gain several orders of magnitude in WIMP detection sensitivity in the near future.

\section{Indirect Detection of Dark Matter}

There is another strategy to look for WIMPs in the galactic halo. This strategy makes use of the fact that neutralinos annihilate mainly into quark anti-quark pairs in the final state, which then fragment into hadrons. The fragmentation products contain stable particles like protons, anti-protons, electrons, positrons, neutrinos, anti-neutrinos and photons. The challenge is to distinguish those particles coming from neutralino annihilations from background particles, which are generated by cosmic ray interactions in the interstellar gas or in the atmosphere. Neutral particles, like photons and neutrinos, have the advantage of carrying directional information in contrast to charged particles which are deflected by the magnetic fields of the 
galaxy. Since the neutralino velocities in the halo are of the order of $10^{-3}$ of the velocity of light, the annihilation can be considered at rest, which means that the energy equivalent to twice the neutralino mass will be distributed amongst the quark anti-quark pair in the final state.

In order to observe an appreciable flux of neutrinos and antineutrinos from neutralino annihilations one has to look at sources where neutralinos are accumulated in rather large concentrations. Such sources can be for example the sun, the earth and the center of our galaxy. They act as gravitational wells into which neutralinos are drawn and where they have accumulated since the time the source was created. The trapping rate of neutralinos within a source, i.e. the sun, depends on their interaction probability with the solar matter and their annihilation probability. Several neutrino telescopes like Kamiokande and SuperKamiokande in Japan, AMANDA at the south pole and ANTARES in the Mediterranean sea looked for neutrinos coming from neutralino annihilations, but the limits they obtained so far are not very strong (Hooper and Silk 2004). A more sensitive experiment ICE CUBE is planned for the future.

Several balloon-borne experiments have been performed to measure anti-protons and positrons from neutralino annihilations. Some experiments have seen an excess of positrons and anti-protons over the expected rate from known sources. However, other experiments did not confirm these findings. In addition, the yield of low energy antiprotons (below $100 \mathrm{MeV}$ ) in cosmic ray interactions in the interstellar gas and in the atmosphere is not well known. This leaves the situation inconclusive for the moment. A discussion and a review of this matter can be found in Bergstrom (2000). However an effort is being made to collect more data on anti-protons and positrons in the near future by two space-borne experiments PAMELA, which has been launched in June this year, and AMS (Anti-Matter Spectrometer) which is still in preparation.

An excess of gamma rays above background (at energies above $1 \mathrm{GeV}$ ) has been observed by the EGRET telescope, which is one of the instruments on the Compton Gamma Ray Observatory. This excess, which was observed in every direction of the galaxy, was studied and interpreted by de Boer (2005) as gamma rays coming possibly from WIMP annihilations in the galactic halo. Assuming a WIMP annihilation at rest the data can be fitted with a WIMP mass of $60 \mathrm{GeV} \mathrm{c}^{-2}$. The authors also claim to be able to reconstruct from these data the peculiar shape of the rotational velocity curve of our galaxy.

The observation of a bright $511 \mathrm{keV}$ gamma ray line by INTEGRAL has been interpreted by Boehm (2004) as a result of low mass (1-100 $\mathrm{MeV} \mathrm{c}^{-2}$ ) WIMP annihilations into electron positron pairs in the galactic bulge. However, the WIMP interpretations of the EGRET and INTEGRAL data will remain subject to a great deal of debates until they can be substantiated, which seems to be inherently difficult with indirect detection methods.

\section{Axion}

The introduction of an axion was not motivated by cosmological considerations but by the fact that the general expression for the QCD Lagrangian contains a term that violates CP. Since the neutron electric dipole moment has so far escaped detection, the coefficient of this term must be tiny. This is referred to as the "strong CP puzzle". Peccei and Quinn (1977) showed that the puzzle can be solved by introducing a neutral pseudoscalar field which neutralizes the $\mathrm{CP}$ violation. This field is associated with a particle, the so-called axion. Axions would be abundantly produced in the early universe by a nonthermal mechanism 
and would qualify for CDM candidates. They are weakly interacting particles which couple to two photons with a coupling strength proportional to their mass $m_{a}$. Although the natural two-photon decay rate would be much too slow $\left(10^{40}\right.$ years) to be detectable, by supplying one of the photons from a coherent source, such as a magnetic field, a significant conversion rate can be achieved. Since the virtual photon from the magnetic field has negligible energy the outgoing real photon carries an energy equal to the sum of the axion rest mass and its kinetic energy. The axion photon conversion of nonrelativistic axions with a mass around $m_{a} \approx 10^{-5} \mathrm{eV} \mathrm{c}^{-2}$ produces photons in the $\mathrm{GHz}$ range. Sikivie (1983) proposed to detect galactic axions by their resonant conversion into photons using a microwave cavity in a strong magnetic field. The power induced by axions in the cavity is very tiny $P \sim V \cdot B^{2} \cdot Q \cdot m_{a} \cdot \rho_{a} \sim 10^{-21} \mathrm{~W}$, with $V$ the volume of the cavity, $B$ the magnetic field and $\rho_{a}$ the axion density. However, since the axion mass is unknown one has to scan over a large range of cavity frequencies with a long integration time at each frequency, which sets practical limits. Fortunately there are constraints which narrow the expected mass range of the axions.

The main constraints on the axion mass come from the stellar evolution and the duration of the supernova SN1987 neutrino signal. Axions can be produced in large quantities in the interior of stars via the Primakoff effect: $\gamma+Z e \rightarrow a+Z e$. In this process a photon interacts with the electric field of a nucleus to produce an axion. Since axions interact very weakly with matter they carry away large amounts of energy. This exotic loss of energy in stars would lead to observable modifications of the standard stellar evolution and is used to constrain the axion mass. Also the duration of the supernova SN1987 neutrino signal provides a limit on the axion mass. After its collapse the supernova core is so hot and dense that neutrinos will be trapped and escape only by diffusion. So it will take several seconds to cool an object of several solar masses. However, the emission of axions would quickly take energy away from the core due to their very weak interactions with matter and would influence the time delay of the observed neutrino signals. Taking all this into consideration we would expect axions to exist in the mass range from $10^{-6} \leq m_{a} \leq 10^{-3} \mathrm{eV} \mathrm{c}^{-2}$.

Pioneering experiments were performed in the 1980s by the University of Rochester, Brookhaven National Laboratory (BNL) and Fermilab (RBF) collaboration and the University of Florida (UF). As shown in Fig. 3, taken from van Bibber and Rosenberg (2006), they reached sensitivities which are still about two orders of magnitude below the KSVZ (Kim, Shifman, Vainshtein, Zakharov) and DFSZ (Dine, Fischler, Srednicki, Zhitnitsky) model predictions. The main difference between the two models is that in the latter axions couple to charged leptons in addition to nucleons and photons. The new generation galactic halo axion experiments employ larger cavity and magnetic field volumes as well as more sophisticated photon detection systems. The Axion Dark Matter Experiment (ADMX) at the Lawrence Livermore National Laboratory (LLNL) is using ultra low noise microwave technology as well as ultra low noise amplifiers. The microwave cavity is inside a 8 Tesla and $4 \mathrm{~m}$ long super-conducting magnet. The experiment of the Kyoto University is pursuing a different technique, namely detecting the photon as a particle rather than a wave. For this they developed a single quantum microwave detection system, which is based on a Rydberg excited atomic beam traversing the cavity. This technique allows a practically noise-free detection of photons (Tada 2006). As shown in Fig. 3 the ADMX experiment has already reached the sensitivity of the model predictions, but within a limited axion mass range. Since the resonance frequencies in the cavities increase with increasing axion mass this method becomes very difficult when searching for axions with masses above $10^{-4} \mathrm{eV} \mathrm{c}^{-2}$. For higher mass axion searches the resonant cavity approach is replaced by experiments using X-ray detection systems. These offer an advantage over the time consuming frequency scanning, since they cover a large mass range at once, but they are much more limited by background. 


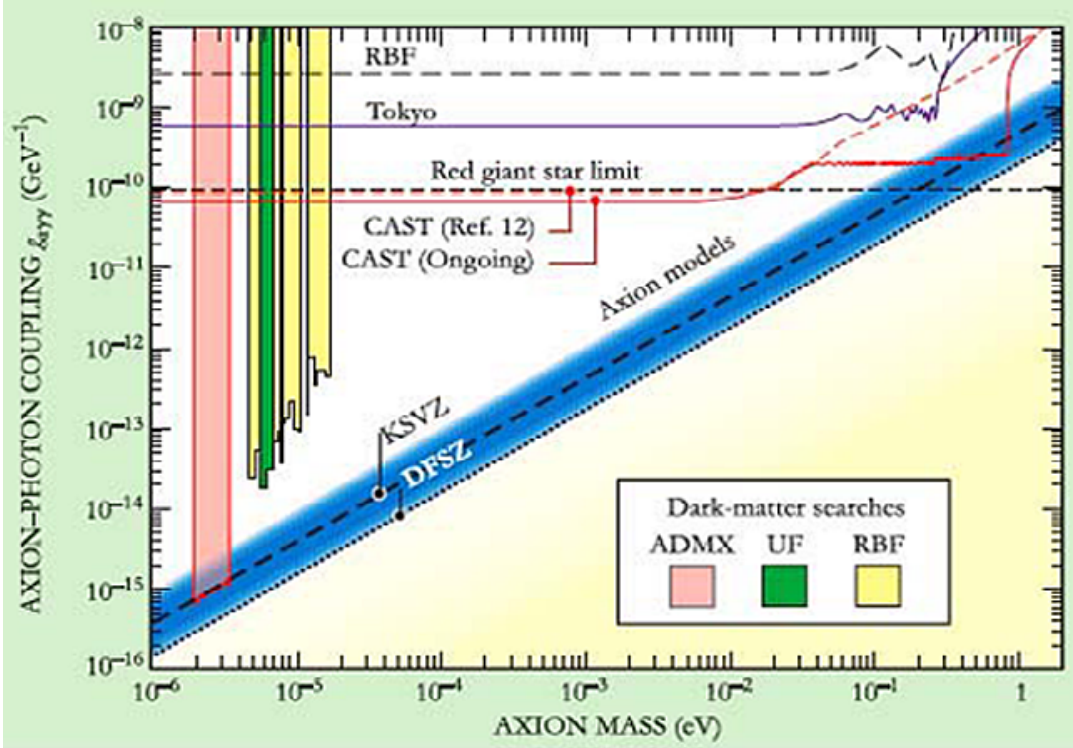

Fig. 3 Exclusion plot of axion-photon coupling versus axion mass

Since axions can be produced in stars, the CAST (CERN Axion Solar Telescope) collaboration has decided to look for axions coming from the sun. Their telescope consists of a $9 \mathrm{~m}$ long, 9 Tesla superconducting dipole magnet (a prototype LHC magnet at CERN) and an X-ray detection system. The mean energy of solar axions is expected to be $4.2 \mathrm{keV}$. The results of phase one of their experiment are shown in Fig. 3 (Zioutas 2005). In the ongoing phase two they have filled the magnet bore with gas $\left({ }^{3} \mathrm{He}\right.$ and $\left.{ }^{4} \mathrm{He}\right)$ with variable pressure in order to increase the mass range of the axions and to reach the sensitivity of the model predictions in the range of 0.1 to $1 \mathrm{eV} \mathrm{c}^{-2}$.

Although no axions have so far been detected by these experiments an intriguing signal has been observed by the PVLAS (Polarization of the Vacuum with Laser) collaboration in Legnaro, Italy. They measured the rotation of the polarization of a laser beam when passing through a magnetic field. Their laser beam passed 44000 times a $1 \mathrm{~m}$ long 5 Tesla magnet. They observed a rotation of $3.9 \times 10^{-12}$ rad per pass (Zavattini 2006). Provided all possible spurious effects can be excluded, the observed signal could find an explanation in the production of axions. However, the inferred mass and coupling of the axion from this observation contradicts the limits reached already by the other above described axion experiments. Further investigations are planned with experiments which are known as "shining the light through a wall" (van Bibber 1987).

\section{Conclusions}

There is compelling evidence that our universe consists of $96 \%$ dark matter/energy of unknown nature. However, with the turning-on of the Large Hadron Collider at CERN in 2007 and the new generation of direct and indirect detection experiments there is hope to bring soon light into the darkness. I would like to conclude with a quotation from Shakespeare: "There is no darkness, only ignorance". 
Acknowledgements I would like to thank the organizers for inviting me to this symposium in honor of Johannes Geiss. I am grateful to Johannes Geiss for many discussions about dark matter and to Rainer Kotthaus for informing me about the latest developments of axion searches and of the CAST experiment.

\section{References}

D.S. Akerib et al., Phys. Rev. D 72, 052009 (2005)

D.S. Akerib et al., Phys. Rev. D 73, 011102 (2006)

G. Angloher et al., Astropart. Phys. 18, 43 (2002)

G. Angloher et al., Astropart. Phys. 23, 325 (2005)

L. Bergstrom et al., Rep. Prog. Phys. 63, 793 (2000)

R. Bernabei et al., Phys. Lett. B 480, 23 (2000)

K. van Bibber et al., Phys. Rev. Lett. 59, 759 (1987)

K. van Bibber, L.J. Rosenberg, Physics Today, Aug. 2006, pp. 30-35

C. Boehm et al., Phys. Rev. Lett. 92, 101301 (2004)

K. Borer et al., Astropart. Phys. 22, 199 (2004)

M. Bravin et al., Astropart. Phys. 12, 107 (1999)

W. de Boer et al., Astron. Astrophys. 444, 51 (2005). See also CERN Courrier Dec. 2005, pp. 17-19

J. Ellis, A. Ferstl, K.A. Olive, Phys. Lett. B 481, 304 (2000)

D. Hooper, J. Silk, New J. Phys. 6, 23 (2004)

G. Jungman et al., Phys. Rep. 267, 195 (1996)

M. Maltoni, T. Schwetz, M. Tortola, J.W.F. Valle, New J. Phys. 6, 122 (2004). See also MINOS hepex/0607088

R.D. Peccei, H.R. Quinn, Phys. Rev. Lett. 38, 1440 (1977)

K. Pretzl, Nucl. Instr. Methods A 454, 114 (2000)

K. Pretzl, Space Sci. Rev. 100, 209 (2002)

V. Sanglard et al., Phys. Rev. D 71, 122002 (2005)

P. Sikivie et al., Phys. Rev. Lett. 51, 1415 (1983). See also Physics Today, Dec. 1996, pp. 22-27

D.N. Spergel et al., 2006. astro-ph/0603449

M. Tada et al., Phys. Lett. A 349, 488 (2006)

S. Yoshida et al., Nucl. Phys. B (Proc. Suppl.) 87, 58 (2000)

E. Zavattini et al., Phys. Rev. Lett. 96, 110406 (2006)

K. Zioutas et al., Phys. Lett. 94, 121301 (2005)

F. Zwicky, Helvetica Physica Acta 6, 110 (1933)

F. Zwicky, Phys. Rev. 51, 67 (1937) 